\title{
Examining the efficacy, safety, and patient acceptability of the etonogestrel implantable contraceptive
}

REVIEW

\section{Heather Hohmann}

Department of Obstetrics, Gynecology, and Reproductive Sciences, University of Pittsburgh School of Medicine, Pittsburgh, PA, USA
Correspondence: Heather Hohmann University of Pittsburgh School of Medicine, Department of Obstetrics, Gynecology, and Reproductive Sciences, Magee-Womens Hospital, 300 Halket Street, Pittsburgh, PA 15213, USA

$\mathrm{Tel}+\mid$ 4I2 64| I44|

Fax +| 41264I II33

Email hhohmann@mail.magee.edu
This article was published in the following Dove Press journal:

Patient Preference and Adherence

17 July 2009

Number of times this article has been viewed

\begin{abstract}
Contraceptive implants provide long-acting, highly effective reversible contraception. The etonogestrel implant (ENG implant) is a single rod implant that offers three years of efficacy. The ENG implant was designed to provide contraceptive efficacy by inhibiting ovulation and Pearl Index scores reported for this method are similar to other long-acting reversible contraception as well as similar to sterilization. The implant has been shown to be safe during breast feeding and may improve symptoms of dysmenorrhea and endometriosis. Irregular bleeding patterns can be expected with the device's use and should be addressed in order to decrease rates of discontinuation.
\end{abstract}

Keywords: contraception, Implanon ${ }^{\circledR}$, progestin-only, ENG-implant

\section{Introduction}

Subdermal contraceptive implants have been studied and used in humans for over twenty years. ${ }^{1}$ Contraceptive implants provide long-acting, highly effective reversible contraception. The most recently introduced subdermal implant, Implanon ${ }^{\circledR}$ (N.V. Organon, Oss, the Netherlands), also referred to as the etonogestrel (ENG) implant, is a single rod implant that offers three years of contraceptive efficacy. ${ }^{2}$ The ENG implant has been used in more than 30 countries, including Australia, Indonesia, and the Netherlands, and was approved by the United States Food and Drug Administration (FDA) in 2006. The ENG implant is an excellent option for women with contraindications to estrogen in addition to any woman who desires long-acting reversible contraception.

The ENG implant is a single rod implant measuring $4 \mathrm{~cm}$ long and $2 \mathrm{~mm}$ in diameter with a solid core of ethylene vinyl acetate (EVA) impregnated with $68 \mathrm{mg}$ of etonogestrel, the biologically active metabolite of desogestrel. ${ }^{3,4}$ The EVA copolymer allows controlled release of hormone over three years of use. ${ }^{5}$ Each implant is provided in a disposable sterile inserter for subdermal application.

\section{Mechanism of action}

The ENG implant was designed to provide contraceptive efficacy by inhibiting ovulation. ${ }^{4}$ Researchers have evaluated for evidence of ovulation in subjects with the ENG implant using both progesterone concentrations and ultrasound. In a study of 32 women, weighing between $80 \%$ and $120 \%$ of ideal body weight, randomized to receive either Implanon ${ }^{\circledR}$, between day 1 and 5 of menses, or Norplant System ${ }^{\circledR}$ (Wyeth, Madison, NJ, USA), between day 1 and 7 of menses, progesterone levels were noted to be above the threshold for ovulation for the first time in two subjects at 30 months in the 
ENG implant users and at 12 months in one Norplant System ${ }^{\circledR}$ user. ${ }^{6}$ Ovulation was confirmed by ultrasound in both subjects at 30 months in the ENG group. In the Norplant System ${ }^{\circledR}$ group ovulation was not confirmed by ultrasound until 18 months of use. Although this study was designed to have subjects complete 36 months of observation, only seven women in the ENG implant group and three women in the Norplant System $^{\circledR}$ group completed the full time course. Additionally, both groups of subjects had estradiol levels decrease to early follicular range after implant insertion. During treatment no subjects had continuously low or high estradiol levels. ${ }^{6}$

Davies and colleagues ${ }^{7}$ demonstrated a similar effect on ovulation suppression during one year of follow up of 15 women. Subjects with a mean age of 32 had a partially leached ENG implant, assuring a hormone release rate of $40 \mu \mathrm{g} /$ day, inserted between day one and five of their menstrual cycle. During the study period, no luteal activity was demonstrated utilizing serum hormone levels or ultrasound. In support of the ENG implants ability to suppress ovulation, this study also demonstrated that the cervical mucous assessment six weeks following implant insertion was significant thickened based on a mean Insler value that had fallen significantly from 13 , pre-insertion, to $3.5(\mathrm{p}=0.0001){ }^{7}$

\section{Pharmacology}

Serum levels of ENG above $90 \mathrm{pg} / \mathrm{ml}$ have been shown to be adequate to prevent ovulation. ${ }^{8}$ Eight hours after insertion, ENG serum levels rise to a mean concentration of $265.9 \pm$ $80.9 \mathrm{pg} / \mathrm{mL}$, with maximum serum concentrations seen by day 4 after insertion, with a variation from day 1 to day $13 .^{6}$ After one year of use the serum concentration decreases to a mean of $196 \mathrm{pg} / \mathrm{mL}^{6,9,10}$ and decreased after three years of use to a mean of $156 \mathrm{pg} / \mathrm{mL}$. ${ }^{3,4}$ Serum levels become undetectable at less than $20 \mathrm{pg} / \mathrm{mL}$ one week after removal. Ovulation will occur in the majority of women within six weeks of ENG implant removal. ${ }^{6}$

After insertion of the ENG implant, estradiol (E2) levels initially decrease to early follicular-phase range. ${ }^{9}$ Following this initial decrease, E2 levels will gradual rise. Given the ENG implants' ability to suppress ovulation, it appears that the pharmacology of the ENG implant creates a situation of anovulation with normal endogenous E2 synthesis. One study of 70 subjects demonstrated that there was not a significant difference between baseline and end-of-study estrogen levels. ${ }^{11}$

\section{Efficacy}

Multiple large trials have demonstrated the high efficacy of the ENG implant. A recently published integrated analysis of 11 international clinical trials with a total of 923 subjects revealed that no pregnancies were reported while the ENG implant was in place. ${ }^{12}$ Six pregnancies occurred with a conception date occurring within the first two weeks after implant removal, yielding a Pearl Index score of 0.27 , 0.30 , and 0.38 per 100 women years of use through years one, two, and three, respectively. ${ }^{12}$ These results support prior published data that found zero pregnancies among 1,716 women who used the implant between two and five years (Pearl Index 0.0 [95\% confidence interval 0.00, 0.09]). ${ }^{9}$ The Pearl Index scores reported for the ENG implant are similar to other long-acting reversible contraception as well as similar to sterilization. The limitation on this data is that efficacy information is not available for women with weights that are above $130 \%$ of ideal weight. The quick return to fertility seen in the ENG users in the integrated analysis may be explained by the pharmacologic data that demonstrates E2 levels during ENG implant use to be in the range of the follicular phase. This E2 level suggests that follicles are present during implant use and thus ovulation can occur rapidly after removal. ${ }^{5,12}$

A multicenter Mexican study followed 417 women during three years of ENG implant use. ${ }^{1}$ A total of 256 women $(61.4 \%)$ completed the full three years of use. Women enrolled in this study had a mean weight of $59.4 \pm 9.3 \mathrm{~kg}$ and a mean body mass index (BMI) of $24.9 \pm 3.9$, with $19.4 \%$ of subjects categorized as overweight (BMI $>25$ ) and $8.9 \%$ categorized as obese $(\mathrm{BMI}>30)$. Once again, no pregnancies were recorded in this study which corresponded to 958.5 women years of observation.

A randomized Chinese study of 200 women directly compared the ENG implant to the six-capsule levonorgestrel (LNG) implant for a four-year period. ${ }^{13}$ Of 153 women who completed the full trial, no pregnancies were reported in either group. This data further supports the ENG implant's effectiveness and suggests that further research may demonstrate that the device has acceptable efficacy for greater than three years of use.

Controlled clinical trials are able to give information about efficacy with perfect use, but information about typical use failure rates is essential for patient counseling. Three years of post-marketing data from Australia demonstrated 218 confirmed pregnancies during ENG implant use when this method was newly introduced to the country. ${ }^{14}$ This data set gives a failure rate of 1.07 per 1000 insertions. Of the cases reported, $21 \%$ of patients were found to have been pregnant prior to the time of implant insertion and $39 \%$ of failures were due to "noninsertion." Although the 
study reports that some physician recognized the failure of insertion, the series did not include the number of providers who failed to make this observation. In total, $21 \%$ of cases had insufficient data to detect the reason for failure and the remaining $19 \%$ of pregnancies were due to method failure. Of the 43 women who experienced a contraceptive failure, eight were determined to be secondary to interactions with other medications, carbamazepine being the most notable. Although no specific studies have examined the ENG implant's interactions with hepatic enzyme-inducing medications, the package insert instructs women to use an additional contraceptive method for at least seven days after stopping such drugs. Until further research is done in this particular area, the post-marketing data suggests that the ENG implant should not be considered first-line contraception for women chronically on these types of medications. Additionally, one failure was in a woman who reported weight gain of greater than $10 \mathrm{~kg}$ between the time of insertion and the pregnancy, thus highlighting the fact that there is limited data about ENG implant efficacy in overweight and obese women.

Results of the above studies demonstrate the ENG implant's excellent efficacy. Even when accounting for the failures noted in the Australian post-marketing surveillance, the implant continues to have one of the highest efficacies of any method available.

\section{Insertion and removal}

The ENG implant is inserted with a pre-loaded single use, sterile applicator. Knowledge and training of the proper insertion and removal techniques are essential for clinical efficacy and for the prevention of complications. The implant is typically inserted in the nondominant arm 6 to $8 \mathrm{~cm}$ above the elbow. At completion of the insertion proper placement of the implant should be done by palpating the patient's arm. ${ }^{15}$ Most studies report insertion times averaging two minutes or less. ${ }^{15}$

Overall, low numbers of ENG implant site complications are reported in the literature. In a large multicenter trial, Croxatto and colleagues ${ }^{2}$ reported a $1.3 \%$ complication rate. Examples cited by the author were having a visible implant tip after insertion and blood loss from the injection site. Additionally, an American study reported a 2.5\% rate of reported intermittent pain at the insertion site over a two-year period of use. ${ }^{16}$

Timing of insertion is dependant on the patient's prior use of contraception. Additionally, a negative pregnancy test should be documented on all patients undergoing ENG implant insertion. For women without preceding hormone use, the ENG implant should be inserted within five days of the start of menses. When switching from a combined oral contraceptive, insertion should occur within seven days of the last active pill. Patients switching from another progestin-only method can have the implant placed at anytime while on the progestin-only pill, at the time of intra-uterine device (IUD) or implant removal, or on the due date of the next contraception injection. Implants may be inserted within five days of a first trimester abortion, within six weeks of a second trimester abortion, or within six weeks of childbirth. ${ }^{3}$

The package labeling indicates that the ENG implant needs to be removed at the end of three years of use. ${ }^{3}$ If the implant is unable to be palpated by the clinician prior to removal, imaging techniques may be necessary prior to proceeding. Case reports have utilized high frequency (10 MHz) ultrasound to detect the acoustic shadow associated with the implant ${ }^{17}$ as well as magnetic resonance imaging as a second line modality if needed. ${ }^{18}$ Once the implant has been located, a 2-3 mm incision is made vertically over the implant under sterile conditions. The rod is than removed utilizing the "pop-out" technique previously described for Norplant System ${ }^{\circledR}$ removal. ${ }^{19}$ Funk and colleagues ${ }^{16}$ published an average removal time of $3.5 \mathrm{~min}$ (range, 0.2-60 $\mathrm{min}$ ) and reported difficulties in only two of 330 removals. This included an implant that broke during removal necessitating a second attempt for complete removal. In a large multicenter study, removal difficulties were reported in $3 \%$ of cases. ${ }^{2}$ The most common reason for difficulty was secondary to implants being placed too deep.

\section{Counseling}

Given the number of contraceptive options available, it is essential that providers focus their efforts on helping women chose the best contraceptive method and providing counseling that helps improve continuation rates of the chosen method. When discussing long-term reversible contraception, physicians are obligated to present all suitable options to their patients and review indications and contraindications for each of the methods. Contraindications to the ENG implant listed on the package insert include known or suspected pregnancy, active venous thromboembolic disease, active liver disease, undiagnosed genital tract bleeding, known or suspected breast cancer, progesterone dependant tumors, or allergy to any of the implants components. ${ }^{3}$ As mentioned above, women chronically using hepatic enzyme-inducing medications may not be good candidates for this method of 
contraception. Patients who are good candidates for this form of contraception are those that desire long-term reversible birth control, have no contraindications to ENG implant use, accept implant insertion and removal, and are ready to accept a change in menstrual bleeding patterns.

\section{Safety and side effect profile Bleeding patterns}

A change in bleeding patterns is common and expected from continuous progestin-only contraceptives. An integrated analysis of 11 international trials which took place in the US, Chile, Asia, and Europe analyzed safety data from a total of 942 subjects. Of subjects included in the analysis of bleeding patterns, $65 \%$ completed the study in which they were enrolled and $35 \%$ of subjects discontinued their study participation early. ${ }^{12}$ Overall $11.1 \%$ of subjects discontinued the ENG implant for bleeding irregularities. As a group, subjects experienced 17.5 days of bleeding and spotting per 90-day reference period. Infrequent bleeding was seen in $33.3 \%$ of reference periods, amenorrhea made up $21.4 \%$ of reference periods, prolonged bleeding made up $16.9 \%$ of reference periods, and frequent bleeding was reported in $6.1 \%$ of all reference periods. Overall the greatest number of bleeding days was seen in the first 90 days of placement and decreased between reference periods 2 and 3 , remaining stable from that time point. Unfortunately, although bleeding patterns were similar between the studies no consistent bleeding pattern could be demonstrated for any individual woman.

A retrospective Swiss study was performed at 12 centers in order to assess acceptability and side-effect profile of the ENG implant. ${ }^{20}$ A total of 1183 women had the ENG implant inserted, of which 991 (84\%) completed one follow-up visit. Mean time from insertion to first follow-up visit was 224 days. Normal bleeding patterns were reported by only $11 \%$ of women. Infrequent bleeding was seen in $28 \%$ of women, where as prolonged bleeding was reported in $15 \%$ of women and metromenorrhagia was reported in $16 \%$ of women. Of women with one follow-up visit, $23.7 \%$ had the implant removed prematurely. The most frequently reported adverse event leading to removal was prolonged and frequent bleeding, comprising $45 \%$ of removals for side effects.

In order to reduce the probability of method discontinuation, different regimes have been examined with the goal of improving bleeding profiles. ${ }^{21-23}$ There is currently only one study that has examined the use of oral medications to reduce the amount and duration and bleeding in ENG implant users. $^{22}$ This study randomized 179 women into one of four treatments. Subjects were women who had used the implant for greater than three months and experienced prolonged or frequent bleeding. Treatments were mifepristone $25 \mathrm{mg}$ taken twice on day 1 followed by four days of placebo, mifepristone $25 \mathrm{mg}$ twice on day 1 followed by four days ethinyl estradiol (EE) $20 \mu \mathrm{g}$ in the morning and placebo at night, doxycycline $100 \mathrm{mg}$ twice a day for five days, or placebo twice a day for five days. Mifepristone combined with EE and doxycycline both significantly reduced bleeding episodes (mean 4.3 days [95\% CI 3.5-5.3] and 4.8 days [95\% CI 3.9-5.8], respectively). ${ }^{22}$ Despite the results of this study, the limited availability of mifepristone in the US decreases the utility of this study's results. Doxycycline may be considered as a method to decrease bleeding episodes in ENG implant users, keeping in mind the risk of side effects.

\section{Dysmenorrhea and endometriosis}

Evidence suggests that dysmenorrhea may be improved in ENG implant users. ${ }^{16,24}$ Simarily, new preliminary data suggests that the therapeutic effect of the ENG implant is similar to depot medroxyprogesterone acetate (DMPA) for the treatment of symptomatic endometriosis. ${ }^{25}$ An American trial demonstrated that among women with a history of dysmenorrhea at baseline, $81 \%$ showed improvement with implant use. ${ }^{16}$ Out of the total subject population, $48 \%$ reported decreased dysmenorrhea with ENG implant use while only $8 \%$ showed an increase in the condition. Similarly, Croxatto ${ }^{24}$ reported a $35 \%$ incidence of dysmenorrhea amongst subjects at baseline with $82 \%$ of these women reporting improvement in symptoms at the end of the study. When complying data from multiple ENG implant studies, differences in bleeding pattern during ENG implant use was not correlated to reported incidence or severity of dysmenorrhea. ${ }^{26}$

A recent open, prospective, randomized pilot study conducted as a noninferiority trial demonstrated that the ENG implant was not worse than DMPA for treatment of histologically proven symptomatic endometriosis. ${ }^{25}$ Using visual analog scale measurements of pain, a $68 \%$ decrease in pain score was seen in the ENG implant group and a 53\% decrease in pain score was seen in the DMPA group after six months of use. Study withdraw rates were similar for each group (four of 21 in the ENG implant group, seven of 20 in the DMPA group). Half of the subjects who withdrew from the ENG implant group reported unbearable bleeding-spotting episodes. The other two subjects withdrew for therapyresistant dysmenorhrea and hot flashes, respectively. Reasons for withdrawal in the DMPA group included hot flashes, pelvic pain resistant to treatment, weight gain, desire to conceive, severe depression, and loss of hair. Overall, of women who 
completed the trial, $57 \%$ of the ENG implant group reported being satisfied or very satisfied with their treatment.

\section{Weight changes}

Weight changes attributed to the use of the ENG implant have been described in a number of clinical trials, although the percentage of women who ultimately have the implant removed for this reason is low. Based on results of a large American trial of the implant over a two-year period, weight increase was reported in about $12 \%$ of subjects, ${ }^{16}$ but only $3.3 \%$ of women withdrew because of this weight increase. The mean increase in BMI from baseline to last measurement was $0.7 \mathrm{~kg} / \mathrm{m}^{2}$. Croxatto and colleagues ${ }^{2}$ reported that approximately $20 \%$ of women reported a greater than $10 \%$ increase in BMI over baseline at one or more measurements. The mean increase in BMI over the study's three-year time period was $3.5 \%$, but the mean change in BMI was only $0.8 \mathrm{~kg} / \mathrm{m}^{2}$, similar to the results from the American trial. Zheng and colleagues ${ }^{13}$ reported a change in body weight in 100 Chinese women using the ENG implant with a breakdown of $0.82 \mathrm{~kg}, 1.15 \mathrm{~kg}$, $2.5 \mathrm{~kg}$, and $3.1 \mathrm{~kg}$ for years $1,2,3$, and 4 of use. Importantly, no women withdrew from the study secondary to weight gain. In a retrospective Swiss study of implant users, 9\% of 991 women at first visit (mean time of 224 days since implant insertion, range 1-677 days) and $9 \%$ of 306 women at the second visit (mean time of 347 days since implant insertion, range 15-709 days) reported weight gain during implant use, but only $7 \%$ of women requesting implant removal reported the primary reason as weight gain..$^{20}$ Lastly, in a retrospective British study following ENG implant use in 324 women, of the 277 for whom information was available, 14 (5\%) women who discontinued implant use within one year cited weight change as their primary reason. ${ }^{27}$ Thus, the overall removal rates because of weight change appear to be in the $3 \%-7 \%$ range in non-Asian populations.

\section{Acne}

In a three year study of 635 women, acne was the second most common (12.6\%) nonbleeding adverse event associated with ENG implant use. ${ }^{24}$ This result is consistent with results from the integrated analysis of international clinical trials in which $11.8 \%$ of drug-related adverse events were from acne. ${ }^{12}$ An American trial of 315 subjects provided baseline and post-ENG implant acne information. ${ }^{16}$ Approximately $26 \%$ of women had acne at baseline and $24 \%$ of women had acne after treatment. From the total population, 16\% reported a decrease from baseline, $70 \%$ reported no change, and 14\% reported increased acne. Of subjects with baseline acne, $61 \%$ reported decreased acne post-treatment and only $7 \%$ reported an increase in acne post-treatment. For those women without acne at baseline, $84 \%$ reported no change and $16 \%$ reported increase in this skin condition.

The results related to acne make it difficult to offer patients clear expectations regarding the incidence or severity of acne while using the ENG implant. Patients should be counseled that there is no apparent trend in regards to acne incidence or improvement while using this method of contraception.

\section{Breast feeding}

The ENG implant appears to be safe to use in breastfeeding women. An open-label nonrandomized comparison study of breastfeeding women using either the ENG implant or a copper IUD for contraception followed 80 women/infant pairs for four months. ${ }^{28}$ Forty-two women chose to use the ENG implant with the remaining 38 women choosing the copper IUD. All women were between ages 18 and 40 and were between 28 and 56 days postpartum at the start of the trial. Overall, no significant difference in total fat, protein, or lactose content of the breast milk was found between the two groups. Importantly, 24-hour milk production and infant growth rates were not different between the two groups. Infants were exposed to the etonogestrel with an initial dose of $19.86 \mathrm{ng} / \mathrm{kg} /$ day, equivalent to $1.7 \%$ of the maternal dose, during the first month of use, but this dose decreased between months 2 and $4 .{ }^{28}$ After completion of this trial, researchers followed women and infants over a three-year period to evaluate any differences in long term outcomes. ${ }^{29}$ Over $80 \%$ of subjects in each group completed this follow up. During this time, there was no difference between the two group's infant growth rates or biparietal head circumferences. Even though the original study was not powered for this outcome, the results further support the initial conclusion of ENG implant safety for breastfeeding women.

\section{Bone mineral density}

Progestin-only hormonal contraception's influence on bone mineral density (BMD) has become an area of controversy since the FDA required the inclusion of a black box warning on the package insert for DMPA. Beerthuizen and colleagues ${ }^{30}$ reported on a comparative study of BMD in users of the ENG implant versus users of nonhormonal IUDs. Forty-four ENG users and 29 IUD users, age 18-40 years, were followed for two years. BMD was measured through the use of dual energy X-ray absortiometry (DEXA) at the lumbar spine, proximal femur, and distal radius. Estradiol levels were comparable 
between the groups at baseline and showed no correlation to baseline BMD. No clinically significant difference was seen in BMD between the ENG implant users and the IUD users. No relationship was noted between estradiol levels and changes in BMD in this study.

A longitudinal three-year trial comparing BMD of the forearm of women using either the ENG implant or the LNG implant found no difference between groups. ${ }^{31}$ At 36 months, both groups had significant decreases in BMD at the distal radius, $-5.9 \%$ in the ENG implant group and $-4.4 \%$ in the LNG implant group, but no difference in the ultradistal radius, where trabecular bone predominates. Although a decrease in BMD was seen in this study, it is important to consider that the BMD in the forearm is not the best predictor of future fracture. Further, there is no long term data that shows that this result has any clinical significance.

\section{Effect on lipid profile and liver function}

In order to evaluate the ENG implants affect on lipid profile, Biswas and colleagues ${ }^{32}$ conducted a randomized trial comparing the ENG implant and the Norplant System ${ }^{\circledR}$ following 80 subjects over two years. ${ }^{32}$ Only three ENG implant users discontinued prior to the study's completion. This study demonstrated that ENG implant users had a significant decrease in serum total cholesterol (TC), highdensity lipoprotein (HDL) cholesterol, and low-density lipoprotein (LDL) cholesterol, which was similar to Norplant System $^{\circledR}$ users. A similar decrease in total cholesterol in $21 \%$ of subjects was noted in an American trial of the ENG implant, ${ }^{16}$ but unlike the results from Biswas and colleagues, ${ }^{32}$ this trial also reported a decrease in triglycerides in 33\% of women using the ENG implant. No significant change in the $\mathrm{HDL} / \mathrm{TC}$ ratio was reported by Biswas and colleagues ${ }^{32}$ but a significant decrease in the HDL/LDL ratio for ENG implant users was seen at one year with a return to pre-insertion level at two years. Despite these results, the change in HDL was only $5.8 \%$ lower at two years compared to baseline and the HDL/LDL ratio was never within a range associated with increase risk of cardiovascular disease. The authors concluded that ENG implant use should not significantly increase the risk of cardiovascular disease.

A case-control study of 18 women followed for 12 weeks after ENG implant placement demonstrated that these subjects had a significant decrease in C-reactive protein, HDL, LDL, cholesterol, testosterone, and sex-binding hormone globulin. ${ }^{33}$ These results lead the authors to conclude that use of this method of contraception does not increase cardiac risk factors in young healthy women. Importantly, manufacturers of the implant recommend following cholesterol values in women with known elevated lipid profiles throughout the use of the implant. ${ }^{3}$

Biswas and colleagues ${ }^{34}$ reported the effects of the ENG implant on liver function tests using the same study design as described above. The authors reported a significant increase in mean total and unconjugated bilirubin in both Norplant System $^{\circledR}$ and ENG implant users. Despite this increase, levels never exceeded the normal range. The researchers did notice an initial significant increase in aspartate transaminase levels in ENG implant users at six months, but after a year of use levels declined toward baseline. Contrary to the above trials, Funk and colleagues ${ }^{16}$ reported no significant change in liver function parameters over a two-year use of the ENG implant. Additionally, Inal and colleagues ${ }^{11}$ found no significant difference between baseline and end-of-study serum glutamic oxalate transaminase, serum glutamic pyruvic transaminase, fasting glucose, HDL, cholesterol, or triglycerides. The mixed results suggest that there may be a small amount of liver dysfunction in ENG implant users. The clinical significance of this in healthy women is unclear. But these changes could have consequences in women with known liver dysfunction.

\section{Conclusion}

The ENG implant provides women with an additional highly effective nonuser-dependant reversible contraceptive option. With greater contraceptive options available, we as providers may be better able to match women's contraceptive needs and desires with an appropriate method. The primary advantage of the ENG implant over other types of contraception is the low rate of contraceptive failure in women who have the implant inserted. The trade-off for women is irregularly irregular bleeding that occurs throughout the lifespan of use. Given the high efficacy of this method, we need to find ways to minimize bleeding issues in order to improve continuation rates.

\section{Disclosure}

The author reports no conflicts of interest in this work.

\section{References}

1. Flores JB, Balderas ML, Bonilla MC, Vazquez-Estrada L. Clinical experience and acceptability of the etonogestrel subdermal contraceptive implant. Int J Gynaecol Obstet. 2005;90(3):228-233.

2. Croxatto HB, Urbancsek J, Massai R, Coelingh Bennink H, van Beek A. A multicentre efficacy and safety study of the single contraceptive implant Implanon. Implanon Study Group. Hum Reprod. 1999;14(4):976-981.

3. Implanon (package insert). Roseland, NJ: Organon USA Inc; 2006.

4. Croxatto HB. Mechanisms that explain the contraceptive action of progestin implants for women. Contraception. 2002;65(1):21-27. 
5. Wenzl R, van Beek A, Schnabel P, Huber J. Pharmacokinetics of etonogestrel released from the contraceptive implant Implanon. Contraception. 1998;58(5):283-288.

6. Makarainen L, van Beek A, Tuomivaara L, Asplund B, Coelingh Bennink H. Ovarian function during the use of a single contraceptive implant: Implanon compared with Norplant. Fertil Steril. 1998; 69(4):714-721.

7. Davies GC, Feng LX, Newton JR, Van Beek A, Coelingh-Bennink HJ. Release characteristics, ovarian activity and menstrual bleeding pattern with a single contraceptive implant releasing 3-ketodesogestrel. Contraception. 1993;47(3):251-261.

8. Diaz S, Pavez M, Moo-Young AJ, Bardin CW, Croxatto HB. Clinical trial with 3-keto-desogestrel subdermal implants. Contraception. 1991;44(4):393-408.

9. Croxatto HB, Makarainen L. The pharmacodynamics and efficacy of Implanon. An overview of the data. Contraception. 1998; 58(6 Suppl):91S-97S.

10. IMPLANON approval letter: US Food and Drug Administration; 2006.

11. Inal MM, Yildirim Y, Ertopcu K, Avci ME, Ozelmas I, Tinar S. Effect of the subdermal contraceptive etonogestrel implant (Implanon) on biochemical and hormonal parameters (three years follow-up). Eur $J$ Contracept Reprod Health Care. 2008;13(3):238-242.

12. Darney P, Patel A, Rosen K, Shapiro LS, Kaunitz AM. Safety and efficacy of a single-rod etonogestrel implant (Implanon): results from 11 international clinical trials. Fertil Steril. 172008.

13. Zheng SR, Zheng HM, Qian SZ, Sang GW, Kaper RF. A randomized multicenter study comparing the efficacy and bleeding pattern of a single-rod (Implanon) and a six-capsule (Norplant) hormonal contraceptive implant. Contraception. 1999;60(1):1-8.

14. Harrison-Woolrych M, Hill R. Unintended pregnancies with the etonogestrel implant (Implanon): a case series from postmarketing experience in Australia. Contraception. 2005;71(4):306-308.

15. Shulman LP, Gabriel H. Management and localization strategies for the nonpalpable Implanon rod. Contraception. 2006;73(4): 325-330.

16. Funk S, Miller MM, Mishell DR Jr, et al. Safety and efficacy of Implanon, a single-rod implantable contraceptive containing etonogestrel. Contraception. 2005;71(5):319-326.

17. Lantz A, Nosher JL, Pasquale S, Siegel RL. Ultrasound characteristics of subdermally implanted Implanon contraceptive rods. Contraception. 1997;56(5):323-327.

18. Westerway SC, Picker R, Christie J. Implanon implant detection with ultrasound and magnetic resonance imaging. Aust $N Z J$ Obstet Gynaecol. 2003;43(5):346-350.

19. Pymar HC, Creinin MD, Schwartz JL. "Pop-out" method of levonorgestrel implant removal. Contraception. 1999;59(6):383-387.

20. Bitzer J, Tschudin S, Alder J. Acceptability and side-effects of Implanon in Switzerland: a retrospective study by the Implanon Swiss Study Group. Eur J Contracept Reprod Health Care. 2004;9(4): 278-284.
21. Witjaksono J, Lau TM, Affandi B, Rogers PA. Oestrogen treatment for increased bleeding in Norplant users: preliminary results. Hum Reprod. 1996;(11 Suppl 2):109-114.

22. Weisberg E, Hickey M, Palmer D, et al. A pilot study to assess the effect of three short-term treatments on frequent and/or prolonged bleeding compared to placebo in women using Implanon. Hum Reprod. 2006;21(1):295-302.

23. Alvarez-Sanchez F, Brache V, Thevenin F, Cochon L, Faundes A. Hormonal treatment for bleeding irregularities in Norplant implant users. Am J Obstet Gynecol. 1996;174(3):919-922.

24. Croxatto HB. Clinical profile of Implanon: a single-rod etonogestrel contraceptive implant. Eur J Contracept Reprod Health Care. 2000; (5 Suppl 2):21-28

25. Walch K, Unfried G, Huber J, et al. Implanon versus medroxyprogesterone acetate: effects on pain scores in patients with symptomatic endometriosis - a pilot study. Contraception. 2009;79(1):29-34.

26. Edwards JE, Moore A. Implanon. A review of clinical studies. $\mathrm{Br} J$ Fam Plann. 1999;24(4 Suppl):3-16.

27. Lakha F, Glasier AF. Continuation rates of Implanon in the UK: data from an observational study in a clinical setting. Contraception. 2006;74(4):287-289.

28. Reinprayoon D, Taneepanichskul S, Bunyavejchevin S, et al. Effects of the etonogestrel-releasing contraceptive implant (Implanon on parameters of breastfeeding compared to those of an intrauterine device. Contraception. 2000;62(5):239-246.

29. Taneepanichskul S, Reinprayoon D, Thaithumyanon P, Praisuwanna P, Tosukhowong P, Dieben T. Effects of the etonogestrel-releasing implant Implanon and a nonmedicated intrauterine device on the growth of breast-fed infants. Contraception. 2006;73(4):368-371.

30. Beerthuizen R, van Beek A, Massai R, Makarainen L, Hout J, Bennink HC. Bone mineral density during long-term use of the progestagen contraceptive implant Implanon compared to a non-hormonal method of contraception. Hum Reprod. 2000;15(1):118-122.

31. Monteiro-Dantas C, Espejo-Arce X, Lui-Filho JF, Fernandes AM, Monteiro I, Bahamondes L. A three-year longitudinal evaluation of the forearm bone density of users of etonogestrel- and levonorgestrelreleasing contraceptive implants. Reprod Health. 2007;4:11.

32. Biswas A, Viegas OA, Roy AC. Effect of Implanon and Norplant subdermal contraceptive implants on serum lipids - a randomized comparative study. Contraception. 2003;68(3):189-193.

33. Merki-Feld GS, Imthurn B, Seifert B. Effects of the progestagen-only contraceptive implant Implanon on cardiovascular risk factors. Clin Endocrinol (Oxf). 2008;68(3):355-360.

34. Biswas A, Biswas S, Viegas OA. Effect of etonogestrel subdermal contraceptive implant (Implanon) on liver function tests - a randomized comparative study with Norplant implants. Contraception. 2004; 70(5):379-382.

35. Hatcher R, Trussell J, Stewart F, et al. Kowal D. Contraceptive Technology. 19th Revised ed. New York: Ardent Media, Inc; 2007.
Patient Preference and Adherence

\section{Publish your work in this journal}

Patient Preference and Adherence is an international, peer-reviewed, open access journal that focusing on the growing importance of patient preference and adherence throughout the therapeutic continuum. Patient satisfaction, acceptability, quality of life, compliance, persistence and their role in developing new therapeutic modalities and compounds to

\section{Dovepress}

optimize clinical outcomes for existing disease states are major areas of interest. This journal has been accepted for indexing on PubMed Central. The manuscript management system is completely online and includes a very quick and fair peer-review system. Visit http://www.dovepress.com/ testimonials.php to read real quotes from published authors. 